\title{
Single-Order Transmission Diffraction Gratings based on Dispersion Engineered All-dielectric Metasurfaces
}

\author{
Shulabh Gupta
}

\begin{abstract}
A single-order transmission diffraction grating based on dispersion engineered all-dielectric metasurfaces is proposed and its wavelength discriminating properties have been theoretically described and confirmed using numerical simulations. The metasurface is designed using a 2D array of all-dielectric resonators, which emulates a Huygens' source configuration to achieve a perfect match to free-space in broad bandwidth. Using a holey dielectric nanodisk structure as the unit cell, the resonant wavelength is tapered across the metasurface to engineer the wavelength dependent spatial phase gradient, to emulate a dispersive prism. Consequently, different wavelengths are steered towards different directions and thus are discriminated on the output image plane. Due to subwavelength periodicities involved, the wavelength discrimination is achieved directly in the zeroth diffraction order of the device, unlike conventional diffraction gratings, thereby providing a high efficiency wavelength discriminating device.
\end{abstract}

\section{INTRODUCTION}

Wavelength discrimination is ubiquitous across the entire electromagnetic spectrum and has widespread applications in communication, instrumentation and ultrafast signal processing applications, such as in spectroscopy [1], imaging [2], wavelength multiplexing and demultiplexing [3][4], to name a few.

While an optical prism is conceptually the most basic wavelength discriminator, the commonly used device in freespace optics is a diffraction grating. A diffraction grating is an optical component with a periodic structure, which splits and diffracts light into several beams travelling in different directions [5]. Among the several space harmonics that are generated, except the zeroth order, all diffractions order exhibit wavelength separation, among which only one of the orders is typically used. The rest of the space harmonics including, in particular, the zeroth order, are the unwanted beams and are the prime reasons for lower efficiencies. While some specialized gratings, such as the blazed gratings, maximize the power in a given order, the achievable efficiency in a chosen order can never be unity due to the unavoidable presence of the zeroth order [6]. An excellent alternative to diffraction gratings, is a virtual image phased array (VIPA) device [7][8]. While they provide fine wavelength resolutions, they exhibit small free-spectral ranges resulting in small bandwidth of operation. More importantly, they always require a collimating cylindrical lens to couple the incident beam inside the structure. Arrayedwaveguide grating (AWGs) is another wavelength separating

S. Gupta is with the Department of Electronics, Carleton University, Ottawa, Ontario, Canada e-mail: shulabh.gupta@carleton.ca device, however they are restricted to integrated optical devices only [9].

In this work, a wavelength separating free-space device based on artificially engineered electromagnetic structures, or metamaterials, is proposed. It discriminates wavelengths in the transmission mode, without generating spurious diffraction orders, or requiring complex feeding setup. The proposed device is based on metasurfaces, which are two dimensional equivalent of volumetric metamaterials [10][11] and have been recently explored for the novel paradigm of flat optical systems [12][13]. While they have been mostly focussed on single wavelength operations, they have also been recently applied towards multi-wavelength broadband devices realizing achromatic lenses [14] and wavelength separators in reflection mode [15][16][17], to name a few. As opposed to the previous works, this work proposes the wavelength separation in the transmission mode. Since light can pass through the grating, transmission gratings can be used in compact inline configurations and thus are ideal for usage in spectrometers and beam splitters in demultiplexing applications. The transmission mode operation thus requires a constraint on the impedance matching of the grating in a wide bandwidth, in addition to maximizing the grating efficiency.

The paper is structured as follows. Sec. 2 summarizes the basic properties of a transmission diffraction grating taking a thin sinusoidal grating as an example. It further explains the principle of the proposed metasurface gratings and provide the theoretical background to construct metasurface grating using Huygens' unit cells. Sec. 3, provides detailed design of the alldielectric metasurfaces and shows numerical simulation results to confirm the operation of the grating. Sec 4 summarizes some features and benefits of the proposed metasurface grating and finally, conclusions are provided in Sec. 5.

\section{PRINCIPLE}

\section{A. Conventional Diffraction Grating}

Let us consider a conventional thin sinusoidal grating with its spatial profile given by

$$
g_{t}(x)=\frac{1}{2}\left[1+m \cos \left(2 \pi \frac{x}{\Lambda}\right)\right]
$$

where $m$ is the modulation depth and $\Lambda$ is the period of the diffraction grating. When such a grating is illuminated with a plane-wave followed by a focussing lens of focal length $d$, the field amplitude in the focal plane of the lens is given by [5] 
$|I(x, y ; d)| \propto\left[\frac{\delta(x)}{2}+\frac{m}{2}\left\{\delta\left(x+\frac{\lambda d}{\Lambda}\right)+\delta\left(x-\frac{\lambda d}{\Lambda}\right)\right\}\right]$.

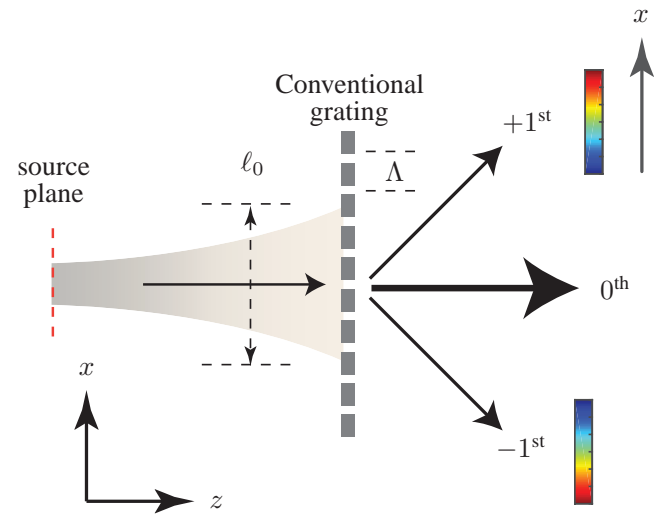

(a)

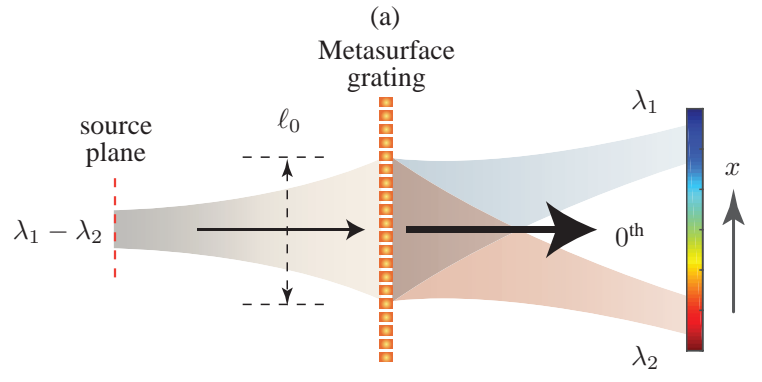

(b)

Fig. 1. Fundamental wavelength discriminating functionality of a) conventional thin sinusoidal diffraction grating, and b) proposed metasurface grating.

The first term corresponds to the zeroth order of the grating and is independent of the wavelength. The last two terms are the $+1^{\text {st }}$ and $-1^{\text {st }}$ diffraction orders, in which light of different wavelengths propagate in different directions. The corresponding wavelength-to-space mapping is given by $x= \pm(\lambda d) / \Lambda$, and this mapping is conventionally used for wavelength separation purposes. These orders are illustrated in Fig. 1 a). Since, only one of the diffraction orders is typically used, the overall diffraction efficiency of the usable order is $(m / 4)^{2}$ resulting in a grating efficiency always less than unity due to other spurious orders.

\section{B. Metasurface Diffraction Grating}

Let us consider again a diffraction grating with a spatial period of $\Lambda$ in the transverse direction along $x$. The spatial frequencies associated with this spatial variation are given by $k_{x}=q(2 \pi / \Lambda)$, where $q \in \mathcal{I}$. The wavenumber of the transmitted wave follows the free-space dispersion, $k_{0}^{2}=$ $k_{x}^{2}+k_{z}^{2}$, and is thus given by

$$
k_{z}=2 \pi \sqrt{\left(\frac{1}{\lambda}\right)^{2}-\left(\frac{q}{\Lambda}\right)^{2}} .
$$

All possible integer values of $q<\Lambda / \lambda$ results in real $k_{z}$ and represent the propagating diffraction orders of the grating.

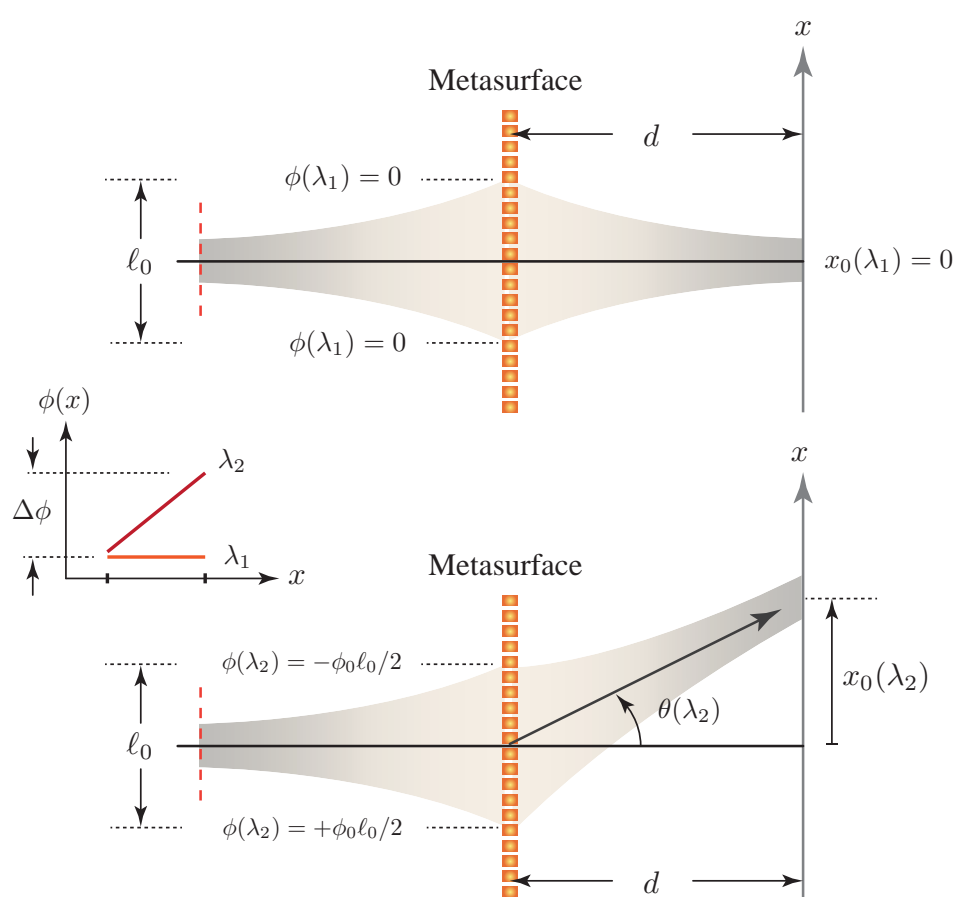

Fig. 2. Principle of the proposed metasurface grating where the metasurface is dispersion engineered to refract different wavelengths towards different angles. The metasurface grating is assumed to be followed by a focal lens which is not shown here for simplicity.

When $\Lambda>\lambda$, there are discrete permissible values of $q$ leading to several diffraction orders. However, when $\Lambda<\lambda$, the only diffraction order allowed by the grating corresponds to $q=$ 0 , i.e. the zeroth order. Therefore, in order to eliminate the higher-order diffraction beams, the grating must have a subwavelength period structure. Metasurfaces are such structures composed of $2 \mathrm{D}$ periodic arrays of sub-wavelength particles and dominantly operate in the zeroth order.

Next consider a non-dispersive metasurface 1 , whose transmission phase is linearly tapered along $x$-axis. The transmittance function of such a metasurface is given by

$$
T(x, y ; \lambda)=\exp \{j \phi(\lambda) x\}=\exp \left\{j \frac{2 \pi \Phi_{0}}{\lambda} x\right\}
$$

where $\Phi_{0}$ is a constant. If the metasurface is followed by a focussing lens of focal length $d$, the intensity distribution at the focal plane of the lens is then given by

$$
|\psi(x, y, d, \lambda)|=\left|\psi(x, y, 0 ; \lambda) T(x, y) h_{\mathrm{lens}}(x, y) * h_{\mathrm{air}}(x, y)\right| .
$$

where $h_{\text {lens }}(x, y)$ and $h_{\text {air }}(x, y)$ are the impulse responses of the lens and the free-space. Assume for simplicity a plane wave excitation so that $\psi(x, y, 0 ; \lambda)=1$. Under paraxial conditions, the output intensity can be shown to be

$$
|\psi(x, y, d ; \lambda)| \propto \delta\left(x-\Phi_{0} d\right)
$$

${ }^{1} \mathrm{~A}$ non-dispersive device is one whose transmission phase is a linear function of frequency $\nu$ or $1 / \lambda$. 
Therefore a non-dispersive metasurface refracts the plane wave and focusses all the wavelengths to $x=\Phi_{0} d$. A non-dispersive metasurface, is thus incapable of wavelength discrimination.

Next, consider a dispersive metasurface with the following property:

$$
\begin{aligned}
\phi\left(\lambda_{1}, x\right)=0 & \Rightarrow T\left(x, y ; \lambda_{1}\right)=1 \\
\phi\left(\lambda_{2}, x\right)=\phi_{0} x & \Rightarrow T\left(x, y ; \lambda_{2}\right)=\exp \left\{j \phi_{0} x\right\}
\end{aligned}
$$

where $\phi_{0}$ is the phase gradient along the $x$-axis, defined as

$$
\phi_{0}\left(\lambda_{2}\right)=\frac{\angle T\left(x, y ; \lambda_{2}\right)}{d x} .
$$

The wave at $\lambda_{1}$ experiences an $x$-independent transmission phase, and thus following (6), focusses at $x=0$, as shown in the upper half of Fig. 2 On the other hand, the wave at $\lambda_{2}$ can be shown to be focussed at

$$
x=\left(\frac{\phi_{0} \lambda_{2} d}{2 \pi}\right),
$$

as shown in the lower half of Fig. 2, and thus can be spatially discriminated from $\lambda_{1}$. Following this example, if the metasurface can be engineered to exhibit a non-zero $\phi_{0}(\lambda)$, different wavelengths will focus onto different $x$-co-ordinates and will form a prism-type rainbow effect on the image plane. The outgoing angle of the wave after the metasurface can be calculated using (10) to obtain the beam scanning law which is given by

$$
\theta(\lambda)=\tan ^{-1}\left(\frac{\phi_{0} \lambda}{2 \pi}\right) .
$$

Dispersion in metasurfaces is thus a necessary condition to achieve wavelength discrimination capability.

\section{Dispersion Engineered Grating based on Lorentz Huygen's Sources}

A metasurface is a 2D array of sub-wavelength particles. It may be modelled by surface polarizabilities, that relate the average electromagnetic fields to the induced dipole moments, i.e. $\mathbf{p}=\hat{\alpha}_{e} E_{\mathrm{av}}$ and $\mathbf{m}=\hat{\alpha}_{m} H_{\mathrm{av}}$, where $E_{\mathrm{av}}$ and $H_{\mathrm{av}}$ are the average electric and magnetic fields tangential to the metasurface, respectively, and $\hat{\alpha}_{e}$ and $\hat{\alpha}_{m}$ are the effective electric and magnetic polarizability densities per unit area [18][19]. It is assumed that $\mathbf{p}$ and $\mathbf{m}$ are oriented perpendicular to each other and in the plane of the metasurface, a configuration that is commonly referred to as the Huygens' source. If $\hat{\alpha}_{m}=\eta_{0}^{2} \hat{\alpha}_{e} \forall \lambda$, where $\eta_{0}$ is the free-space impedance, it can shown that the metasurface is perfectly matched to free space and its transfer function is given by [18][19]

$$
T_{\mathrm{m}}(\lambda)=\left[\frac{\lambda+i 2 \pi c \eta_{0} \hat{\alpha}_{\mathrm{e}}(\lambda) / 2}{\lambda-i 2 \pi c \eta_{0} \hat{\alpha}_{\mathrm{e}}(\lambda) / 2}\right] .
$$

If Lorentz dispersion is further assumed for $\hat{\alpha}_{e}$, given by

$$
\hat{\alpha}(\lambda)=\frac{\lambda_{p}^{2}}{\lambda_{r}}\left[\frac{\lambda_{r}}{\lambda_{r}^{2}-\lambda^{2}}-\frac{j \pi}{2}\left\{\delta\left(\lambda+\lambda_{r}\right)+\delta\left(\lambda-\lambda_{r}\right)\right\}\right],
$$

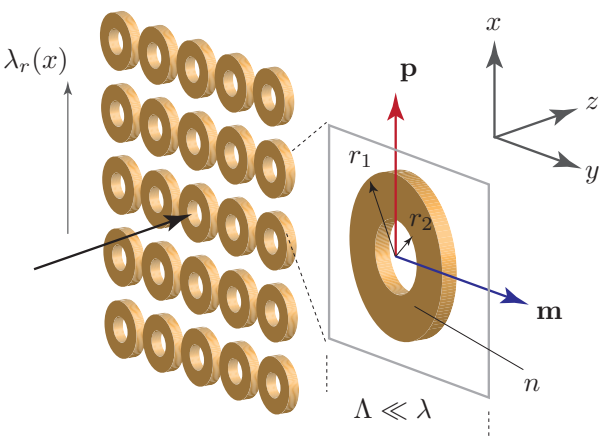

(a)
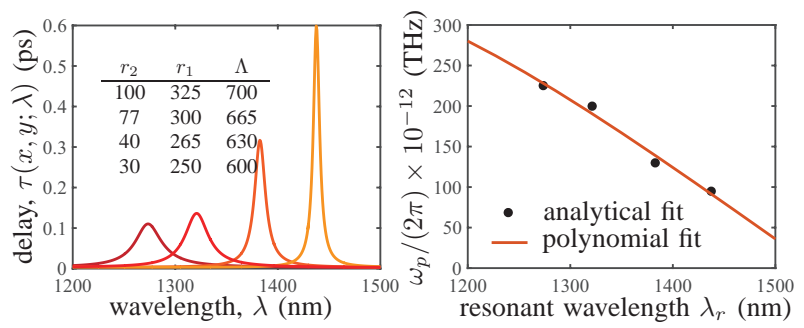

(b)

Fig. 3. Dispersion engineered all-dielectric metasurfaces. a) Metasurface unit cell. b) FEM-HFSS simulated dispersion response of 4 different unit cells using periodic boundary conditions, and the extracted $\lambda_{p}$ vs $\lambda_{r}$ relation using analytical transfer function of 14. Here $n=3.5, n_{0}=1.66$ and $t=220 \mathrm{~nm}$.

where $\lambda_{r}$ is the resonant wavelength of the particles and $\lambda_{p}$ is the plasma wavelength, the general transfer function of (12) becomes

$$
T(\lambda)=\left[\frac{\lambda\left(\lambda_{r}^{2}-\lambda^{2}\right)+j \pi \eta_{0} c \lambda_{p}^{2}}{\lambda\left(\lambda_{r}^{2}-\lambda^{2}\right)-j \pi \eta_{0} c \lambda_{p}^{2}}\right]=\exp \{j \phi(\lambda)\}
$$

noting that under these assumptions, the metasurface acts as an all-pass surface, with $|T(\lambda)|=1 \forall \lambda$. Its corresponding wavelength dependent transmission phase is thus given by

$$
\phi(\lambda)=2 \tan ^{-1}\left[\frac{\pi \eta_{0} c \lambda_{p}^{2}}{\lambda\left(\lambda_{r}^{2}-\lambda^{2}\right)}\right] .
$$

The dispersion of the metasurface is therefore controllable through the resonant wavelength parameter, $\lambda_{r}$. It should be noted that in a physical resonator, the resonant wavelength $\lambda_{r}$ and the plasma wavelength $\lambda_{p}$ are typically related, and $\lambda_{r}$ is the only free design parameter. A metasurface constructed using Lorentz-type Huygens' sources, thus provide an ideal framework to engineer the dispersion of the metasurface, while ensuring a perfect match in a broad bandwidth.

\section{Metasurface Grating Demonstration}

A Lorentz metasurface is typically implemented using a 2D array of all-dielectric resonators in the form of cylindrical nanodisks, which naturally provide the Huygens' source configuration of orthogonal $\mathbf{p}$ and $\mathbf{m}$, considered herein, providing the required transfer function of (14) [20][21]. Fig. 3 (a) shows an example of an all-dielectric metasurface, which is particularly suitable for engineering the metasurface dispersion. The unit 
cell of size $\Lambda<\lambda$, is composed of a nanodisk of radius $r_{1}$ and $r_{2}$ made of a material with a refractive index $n$ which could be buried in a host dielectric of refractive index $n_{0}$. Radii $r_{1}, r_{2}$ and the unit cell size $\Lambda$, control the resonant wavelength $\lambda_{r}$ of the metasurface, for a given index $n$ and disk thickness $t$, and thus can be designed to engineer the metasurface dispersion.

Fig. 3(b) shows the typical dispersion (delay) response, obtained using periodic boundary conditions in FEM-HFSS, for varying physical parameters of the metasurface unit cell formed using silicon nanodisks with $n=3.5$ buried in a host dielectric of refractive index $n_{0}=1.66$ of thickness $t=220 \mathrm{~nm}$. The bandwidth chosen here for demonstration is between 1200-1500 nm. As can be seen, the resonant wavelength, where the delay is maximum, can be conveniently varied over a large bandwidth, ensuring a near perfect match in the entire bandwidth $(20 \log |T(\lambda)|<-10 \mathrm{~dB}$, not shown here). It is also noticed that as $\lambda_{r}$ increases, the delay swing increases resulting in a stronger dispersion.

The transmission response of each nano-disk unit cell is then analytically fitted using the Lorentz transfer function of (14) to extract the corresponding plasma wavelength $\lambda_{p}$. The resulting plot of $\lambda_{p}$ vs $\lambda_{r}$ is also shown in Fig. 3 (b) which is then used to obtain a continuous distribution using polynomial fitting. This polynomial function thus acts as a look-up function to realize a continuous sweep of $\lambda_{r}$ across the entire bandwidth.

Next, a metasurface is dispersion engineered by sweeping $\lambda_{r}$ linearly along the $x$-axis, to create a non-zero phase gradient $\phi_{0}$, following the relationship:

$$
\lambda_{r}(x, y)=\lambda_{\min }+\left(x+\ell_{0} / 2\right) \frac{\lambda_{\max }-\lambda_{\min }}{\ell_{0}} .
$$

The typical transmission phase distribution of the metasurface is shown in Fig. 4 a) as function of the wavelength and the spatial co-ordinate $x$. The metasurface is $\ell_{0}^{2}=100 \mu \mathrm{m} \times$ $100 \mu \mathrm{m}$ in size. As clearly seen in the figure, the phase gradient $\phi_{0}$ is a function of wavelength. Since $\phi_{0}$ is also a function of $x$, to obtain the scan law $\theta(\lambda)$ of the metasurface, $\phi_{0}$ is evaluated at the centre of the metasurface at $x=0$. Fig. 4(b) shows the obtained scanning law of this metasurface. The scanning law is strongly nonlinear and thus has varying wavelength resolution depending on the wavelength, which becomes finer near $1300 \mathrm{~nm}$, i.e. large change in $\theta$ for a small change in $\lambda$. This is due to the fact that the phase gradient parameter depends on wavelength as opposed to a desired constant value. Further, the scan possesses a symmetry around $1300 \mathrm{~nm}$ enabling two separate bands of operation with identical wavelength resolutions. The metasurface can thus be either operated in 1200-1300 nm band or 1300-1400 nm band. In each band, the angular dispersion of the grating $\approx 0.8^{\circ} / 50 \mathrm{~nm}$.

Figure. 5 shows the computed field distribution at different wavelengths when the metasurface of Fig. 4 is incident with a Gaussian beam. The fields are computed using analytical Fourier propagation such that $\psi(x, y, z ; \lambda)=$ $\psi(x, y, z ; \lambda) T(x, y, z ; \lambda) * h_{\text {air }}(x, y, z ; \lambda)$. As expected, different wavelengths propagate in different direction after hitting the metasurface following the scanning law, and thus form a spectral rainbow at the output image planes. This demonstrates
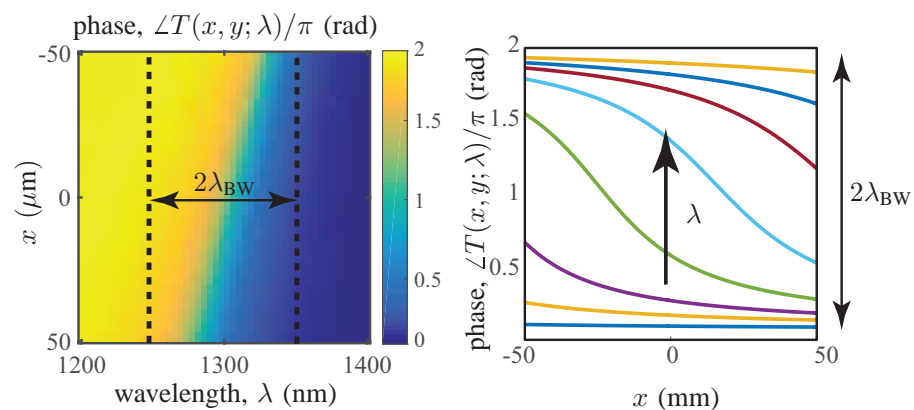

(a)
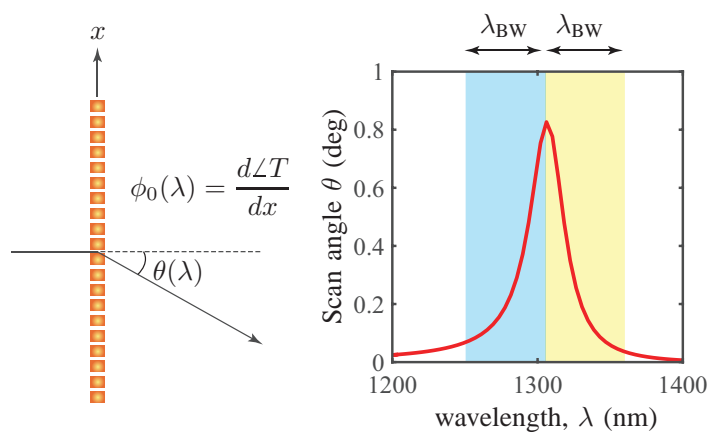

(b)

Fig. 4. Metasurface grating characteristics. a) Metasurface transmission phase as a function of wavelength along the $x$-axis of the metasurface. $\mathrm{b}$ ) Wavelength scanning law. Here $\lambda_{\max }=1325 \mathrm{~nm}$ and $\lambda_{\min }=1275 \mathrm{~nm}$ and $\ell_{0}=100 \mu \mathrm{m}$.

the wavelength separation capability of the designed alldielectric metasurface grating.

\section{FeATURES AND Benefits}

1) The metasurface grating can be seen as a flat prism, which is engineered on an "atomic" scale to achieve wavelength separation with $100 \%$ transmission. Dispersion engineering at the unit scale dimensions, provides a highly flexible grating design, adaptable for specified bandwidths and wavelength resolution requirements.

2) Similar to optical prisms, the proposed metasurface grating operates in the fundamental zeroth order diffraction mode. Compared to conventional diffraction gratings, no energy is wasted in the higher orders in the met surface gratings, thereby making it possible to theoretically achieve a maximum efficiency of $100 \%$.

3) The all-dielectric metasurface implementation based on Huygen's sources ensures a perfect match to free-space which is a desirable feature for such a transmissionmode device.

4) Compared to the typically used plasmonic unit cells, the dielectric unit cells employed in the metasurface design herein, is ideal for optical frequencies offering low dissipation losses, to help maximize the grating efficiency.

\section{CONCLusions}

A single-order transmission diffraction grating based on dispersion engineered all-dielectric metasurfaces has been 

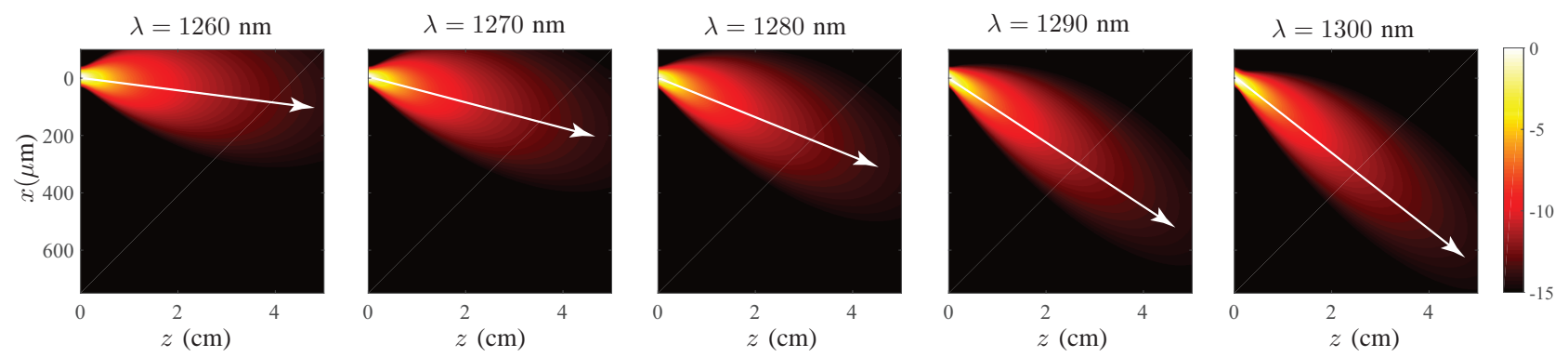

Fig. 5. The analytical field distribution in the $x-z$ plane when a gaussian beam of width $w_{0}$ is incident on the dispersion engineered metasurface of Fig. 4 for various different wavelengths. The Gaussian beam assumed is $\psi(x, y, 0)=\exp \left\{-\left(x^{2}+y^{2}\right) / 2 w_{0}^{2}\right\}$.

proposed. It's wavelength discriminating properties have been theoretically described and its operation has been confirmed using numerical simulations. The metasurface has been designed using all-dielectric unit cell, which emulates a Huygens' source configuration to achieve a perfect match to freespace in broad bandwidth. Using a holey dielectric nanodisk structure as the unit cells, the resonant wavelength is varied across the metasurface to engineer the wavelength dependent spatial phase gradient, required for achieving the wavelength discrimination. Finally, a numerical demonstration has been provided to achieve an angular dispersion of about $0.8^{\circ} / 50 \mathrm{~nm}$ within 1250-1300 $\mathrm{nm}$ bandwidth. Its experimental validation is currently under progress and will be reported elsewhere.

\section{ACKNOWLEDGEMENT}

The author would like to thank Dr. Fangxin Li, from Synaptive Medicals, Toronto, for fruitful discussions related to diffraction gratings and state-of-the art spectrum analyzers.

\section{REFERENCES}

[1] D. R. Solli, J. Chou, and B. Jalali, "Amplified wavelength-time transformation for real-time spectroscopy," Nature Photonics, no. 2, pp. 48-51, Dec 2007.

[2] P. Metz, J. Adam, M. Gerken, and B. Jalali, "Compact, transmissive twodimensional spatial disperser design with application in simultaneous endoscopic imaging and laser microsurgery," Appl. Opt., vol. 53, no. 3, pp. 376-382, Jan 2014.

[3] S. Xiao and A. M. Weiner, "2-D wavelength demultiplexer with potential for $\geq 1000$ channels in the C-band," Opt. Express, vol. 12, no. 13, pp. 2895-2902, Jun 2004

[4] K. Goda and B. Jalali, "Dispersive fourier transformation for fast continuous single-shot measurements," Nature Photonics, vol. 7, pp. 102-112, Jan 2013.

[5] J. Goodman, Introduction to Fourier Optics. Roberts and Company Publishers; 3rd Edition edition, 2004.

[6] E. G. Loewen, M. Nevière, and D. Maystre, "Grating efficiency theory as it applies to blazed and holographic gratings," Appl. Opt., vol. 16, no. 10, pp. 2711-2721, Oct 1977.

[7] M. Shirasaki, "Large angular dispersion by a virtually imaged phased array and its application to a wavelength demultiplexer," Opt. Lett., vol. 21, no. 5, pp. 366-368, Mar 1996.

[8] A. Vega, A. M. Weiner, and C. Lin, "Generalized grating equation for virtually-imaged phased-array spectral dispersers," Appl. Opt., vol. 42, no. 20, pp. 4152-4155, Jul 2003.

[9] B. E. A. Saleh and M. C. Teich, Fundamentals of Photonics, 2nd ed. Wiley-Interscience, 2007.

[10] A. V. Kildishev, A. Boltasseva, and V. M. Shalaev, "Planar photonics with metasurfaces," Science, vol. 339, no. 6125, 2013

[11] C. Holloway, E. F. Kuester, J. Gordon, J. O'Hara, J. Booth, and D. Smith, "An overview of the theory and applications of metasurfaces: The twodimensional equivalents of metamaterials," Antennas and Propagation Magazine, IEEE, vol. 54, no. 2, pp. 10-35, April 2012.
[12] N. Yu and F. Capasso, "Flat optics with designer metasurfaces," Nature Materials, vol. 13, April 2014.

[13] — "Optical metasurfaces and prospect of their applications including fiber optics," J. Lightwave Technol., vol. 33, no. 12, pp. 2344-2358, Jun 2015.

[14] F. Aieta, M. A. Kats, P. Genevet, and F. Capasso, "Achromatic metasurface optical components by dispersive phase compensation," arXiv:1411.3966, Nov. 2014.

[15] Z. Li, E. Palacios, S. Butun, and K. Aydin, "Visible-frequency metasurfaces for broadband anomalous reflection and high-efficiency spectrum splitting," Nano Letters, vol. 15, no. 3, pp. 1615-1621, 2015.

[16] J. Chen, F. Gou, Y. Wu, M. Zhang, and Z. Zhang, "Beam steering with nanoring reflectarray metasurfaces," in 14th IEEE International Conference on Nanotechnology, Aug 2014, pp. 333-336.

[17] L. Zhu, J. Kapraun, J. Ferrara, and C. J. Chang-Hasnain, "Flexible photonic metastructures for tunable coloration," Optica, vol. 2, no. 3, pp. 255-258, Mar 2015.

[18] K. Achouri, M. Salem, and C. Caloz, "General metasurface synthesis based on susceptibility tensors," IEEE Trans. Antennas Propag., vol. 63, no. 7, pp. 2977-2991, July 2015.

[19] C. Pfeiffer and A. Grbic, "Metamaterial huygens' surfaces: Tailoring wave fronts with reflectionless sheets," Phys. Rev. Lett., vol. 110, p. 197401, May 2013. [Online]. Available: http://link.aps.org/doi/10.1103/PhysRevLett.110.197401

[20] S. Jahani and Z. Jacob, "All-dielectric metamaterials," Nature Nanotechnology, vol. 2, no. 11, pp. 23-36, Jan 2016.

[21] M. Decker, I. Staude, M. Falkner, J. Dominguez, D. N. Neshev, I. Brener, T. Pertsch, and Y. S. Kivshar, "High-efficiency dielectric huygens surfaces," Advanced Optical Materials, vol. 3, no. 6, pp. 813-820, 2015. [Online]. Available: http://dx.doi.org/10.1002/adom.201400584 